\title{
A Novel Approach for Solving Quadratic Fractional Programming Problems
}

\author{
Mustafa Sivri $^{1}$, Inci Albayrak ${ }^{1, *}$ and Gizem Temelcan ${ }^{2}$ \\ ${ }^{1}$ Department of Mathematical Engineering, Yildiz Technical University, Davutpasa, 34220 Esenler, Istanbul, \\ Turkey \\ E-mail: <\{msivri,ibayrak\}@yildiz.edu.tr> \\ ${ }^{2}$ Department of Computer Programming, Istanbul Aydin University, Florya Campus, Kucukcekmece, \\ Istanbul, Turkey \\ E-mail: <temelcan.gizem@gmail.com>
}

\begin{abstract}
In this paper, quadratic fractional programming (QFP) problems involving a factorized or non-factorized objective function and subject to homogenous or non-homogenous constraints are considered. Our proposed approach depends on a computational method that converts the QFP problem into a linear programming (LP) problem by using a Taylor series to solve the problem algebraically. This approach, based on the solution of LP problems, can be applied to various types of nonlinear fractional programming problems containing nonlinear constraint(s), and minimizes the total execution time on iterative operations. To illustrate the solution process, two examples are presented and the proposed approach is compared with other two existing methods for solving QFP problems.
\end{abstract}

Keywords: linear programming problem, quadratic fractional programming problem, Taylor series

Received: February 9, 2018; accepted: September 9, 2018; available online: December 13, 2018

DOI: 10.17535 /crorr.2018.0015

\section{Introduction}

Fractional programming (FP) problems (also known as ratio optimization problems) are optimization problems in which the ratio of two given functions subject to given constraints is to be maximized or minimized. The FP problem has an important reputation in operational research regarding its applications. FP problems are useful in financial and corporate planning, production planning, traffic planning, healthcare and hospital planning, network flows, game theory, etc. Many algorithms have been developed to solve various forms of FP problems.

Considering applications of FP problems, the following classifications can be presented. The Single-Ratio Linear FP Problem is given as:

\footnotetext{
* Corresponding author.

http://www.hdoi.hr/crorr-journal
}

(C2018 Croatian Operational Research Society 


$$
\operatorname{Min} \frac{f_{1}(x)}{f_{2}(x)}
$$

where $\Omega=\left\{x: l_{r}(x) \leq 0, r=1, \ldots, n\right\}, f_{2}(x)>0$ for all $x \in \Omega$ and $l_{r}: x \rightarrow R(r=1, \ldots, n)$, and $f_{1}, f_{2}$ and $l_{r}$ are affine functions.

The Single-Ratio FP Problem is given as:

$$
\operatorname{Min} \frac{f_{1}(x)}{f_{2}(x)}
$$

where $f_{1}$ and $f_{2}$ are extended real-valued functions which are finite-valued on $\Omega$, and $f_{2}(x)>0$ for all $x \in \Omega$, and $\Omega$ is a non-empty closed feasible region in $X$.

The Single-Ratio QFP Problem is given as:

$$
\operatorname{Min} \frac{f_{1}(x)}{f_{2}(x)}
$$

where $f_{1}$ and $f_{2}$ are quadratic, $f_{2}(x)>0$ for all $x \in \Omega$, and $l_{r}: x \rightarrow R(r=1, \ldots, n)$ is an affine function.

The Generalized FP Problem is given as:

$$
\operatorname{Min}\left(\sup _{1 \leq i \leq m} \frac{f_{i}(x)}{g_{i}(x)}\right)
$$

with extended real-valued functions $f_{i}, g_{i}: X \rightarrow(-\infty, \infty)$ which are finite-valued on $\Omega$ with $g_{i}(x)>0$ for all $i=1, \ldots, m$ and $x \in \Omega$.

The Min-Max FP Problem is given as:

$$
\operatorname{Min}_{x \in \Omega} \operatorname{Max}_{y \in \Theta} \frac{f_{1}(y, x)}{f_{2}(y, x)}
$$

where $\Omega \subset R^{m}$ and $\Theta \subset R^{n}$ are non-empty closed sets, and $f_{1}: R^{m+n} \rightarrow(-\infty, \infty)$ is a finitevalued function on $\Omega \times \Theta$. In this case $f_{2}: R^{m+n} \rightarrow(-\infty, \infty)$ is a finite-valued positive function on $\Omega \times \Theta$.

The Summation of Ratio FP Problem is given as:

$$
\operatorname{Min} \sum_{i=1}^{m} \frac{f_{i}(x)}{g_{i}(x)}
$$

with $g_{i}(x)>0$ for all $i=1, \ldots, m$ and $x \in \Omega$.

The Multi-Objective FP Problem is given as:

$$
\operatorname{Min}\left(\frac{f_{1}(x)}{g_{1}(x)}, \frac{f_{2}(x)}{g_{2}(x)}, \ldots, \frac{f_{m}(x)}{g_{m}(x)}\right)
$$

with $g_{i}(x)>0$ for all $i=1, \ldots, m$ and $x \in \Omega$ [7]. 
Schaible and Shi [18] reviewed recent developments in single-ratio and generalized (max-min) FP problems. Linear Fractional Programming (LFP) problem is a kind of nonlinear programming problems in which the nominator and the denominator of the objective function, and the constraints, are linear functions. To find the optimal solution of an LFP problem, different solution algorithms have been developed in the literature. In 1956, Isbell and Marlow [10] found the optimal solution to an LFP problem by using a sequence of LP problems; in 1962, Charnes and Cooper [4] developed an algorithm replacing the LFP problem with LP problems by transforming the variables. A common feature of these algorithms is that the optimal solution of the LFP problem is determined without providing the decision maker with extra information about the solution process. Swarup [23] extended the simplex technique for LP problems and dealt with the problem of maximizing the ratio of two linear functions under constraints of linear equalities and the non-negativity of the variables. Bitran and Novaes [3] proposed an algorithm based on a simplex routine for solving a sequence of LP problems, which depends on updating the local gradient of the fractional objective function at successive points. Bitran and Novaes [3] considered the objective function as the ratio of two linear functions. Bitran and Magnanti [2] considered duality and sensitivity analysis in LFP problems. Wolf [28] proposed a parametric method for determining the optimal solution of the LFP problem that was different from the usual algorithms. Tantawy [25] developed an iterative method based on the conjugate gradient projection method. In this paper, the feasible direction approach moving through the feasible region improves the objective function of the LFP problem. Tantawy [24] also developed an iterative method that can be used for sensitivity analysis to solve LFP problems. Pramanik, Dey, and Giri [16] used a fuzzy goal programming approach, and solved multi-objective linear plus LFP problems based on Taylor series approximation. Effati and Pakdaman [6] proposed a method to solve an interval-valued LFP problem. Odior [14] developed an approach for when the constraints of the LFP are in the form of an inequality in which the objective function is a linear fractional function. Pandian and Jayalakshmi [15] presented a new approach, which is called the denominator objective restriction method and is based on the simplex method for solving LFP problems. Tantawy and Sallam [27] presented a new method for solving integer LFP problems when the objective function is linear fractional and the constraints are in the form of linear inequalities, and then Tantawy [26] proposed a duality approach to solve LFP problems in the same form as Tantawy [24]. Saha, Hossain, Uddin, and Mondal [17] developed a new technique by converting the LFP problem into a single LP problem for some cases of the nominator and denominator function. Simi and Talukder [20] presented a new approach for transforming the LFP problem into an LP problem and solving the problem using the duality.

As a special type of optimization problem, QFP problems have many different applications in operations research literature. These types of problems can be classified on the basis of the homogeneity of the constraints and the factorability of the objective function. Singh [21] established the optimality conditions of Kuhn-Tucker and Fritz John without differentiability for nonlinear FP problems. Gotoh and Konno [8] solved the general QFP problem globally by combining the Dinkelbach method and a branch and bound approach. Khurana and Arora [12] developed an approach for solving QFP problems in which some of the constraints are homogenous. This alternative method transforms the original problem to a new 
formulation that has fewer constraints but more variables. Sharma and Singh [19] proposed a new approach for solving factorized QFP problems and linear factorized QFP problems based on the iterative procedure of simplex techniques. Suleiman and Nawkhass [22] recommended that a modified simplex method is superior in addressing QFP problems, and used Wolfe's method for solving QFP problems. Hasan and Hasan [9] solved a QFP problem with homogenous constraints and a factorized fractional objective function that cannot be solved directly by the extended simplex method. Abdulrahim [1] developed a convergent algorithm for the extreme point of the QFP problem, and used a new cutting plane technique to obtain the optimal solution. Nguyen, Sheu, and Xia [13] considered minimizing a single-ratio QFP problem subject to two-sided quadratic constraints. Jayalakshmi [11] presented a new approach, which is called the decomposition fractional separable method and is based on the LP problem in which the numerator and denominator of the objective function can be factorized into two linear functions. Youness, Maaty, and Eldidamony [29] introduced a two-dimensional algorithm based on a parametric approach for finding the solutions of nonlinear fractional optimization problems.

In this paper, a new approach is presented for finding the solution of the QFP problems involving a factorized or non-factorized objective function and subject to homogenous or nonhomogenous constraints. To solve any QFP problem, our proposed approach easily converts the problem into an LP problem by using a Taylor series at a chosen initial point. The objective function of the QFP problem is expanded to a Taylor series using our approach. The proposed approach obtains successive LP problems, and determines an optimal solution of the QFP problem. The proposed approach has computationally faster steps, and can be applied more easily than other algebraic methods. Since the final solution can be obtained rapidly, this approach will also be suitable for computer use.

This paper is organized as follows. Section 2 briefly presents the required information used in this study. In Section 3, the proposed approach is demonstrated. Section 4 and Section 5 consist of numerical examples and the conclusion, respectively.

\section{Preliminaries}

Definition 1. A QFP problem is defined as

$$
\text { Optimize } z(x)=\frac{f(x)}{g(x)}
$$

subject to

$$
\begin{aligned}
& A x(\leq,=, \geq) B \\
& x \geq 0
\end{aligned}
$$

where $x$ is an $n$-dimensional column vector of decision variables, $A$ is an $(m \times n)$ matrix and $B$ is an $m$-dimensional column vector of constants.

Definition 2[5]. Any point $x$ satisfying the constraints (1.b) and (1.c) is called a feasible point. The set of all feasible points is called the feasible set, such that $X=\left\{x \in R^{n}: A x(\leq,=, \geq) B ; x \geq 0\right\}$. 
Definition 3. A feasible point $x^{*}$ is called the optimal solution to the optimization problem (1) if $z(x) \leq z\left(x^{*}\right)$ for the maximization problem $\left(z(x) \geq z\left(x^{*}\right)\right.$ for the minimization problem) for each feasible point $x$.

Definition 4. A differentiable function $F$ is continuously differentiable if and only if $F$ is of differentiability class $C^{1}$.

Definition 5. The first two terms of the Taylor series generated by $F\left(x_{1}, \ldots, x_{n}\right)$ at $A=\left(a_{1}, \ldots, a_{n}\right)$, i.e.

$$
F(A)+\frac{\partial}{\partial x_{1}} F(A)\left(x_{1}-a_{1}\right)+\frac{\partial}{\partial x_{2}} F(A)\left(x_{2}-a_{2}\right)+\ldots+\frac{\partial}{\partial x_{n}} F(A)\left(x_{n}-a_{n}\right)=0
$$

linearize the function $F$ in $n$ variables.

\section{The proposed approach}

Consider the QFP problem given in (1). Our algorithm is given as follows:

Step 1. Choose any initial arbitrary feasible non-zero point, i.e. $\breve{x}=\left(\breve{x}_{1}, \ldots, \breve{x}_{n}\right)$.

Step 2. Linearize the objective function (1.a) by expanding it to the Taylor series at the chosen point $\breve{x}$ using Definition 5 .

Step 3. Solve the following LP problem:

$$
\text { Opt } z_{L}(x)=C x
$$

subject to

$$
\begin{gathered}
A x(\leq,=, \geq) B \\
x \geq 0
\end{gathered}
$$

where the subscript $L$ is used to show the linearization of the objective function (1.a) obtained in Step 2, and the constraints are the same as in (1.b) and (1.c). Solve this LP problem, and find a solution $\bar{x}=\left(\bar{x}_{1}, \ldots, \bar{x}_{n}\right)$.

Step 4. Expand the objective function (1.a) to the Taylor series at the obtained solution $\bar{x}$.

Step 5. Solve the reconstructed LP problem consisting of the linearized objective function at $\bar{x}$ obtained in Step 4 and subject to (1.b) and (1.c), and find another solution $\underline{x}=\left(\underline{x}_{1}, \ldots, \underline{x}_{n}\right)$.

Step 6. Check two successive solutions:

- If the solutions $\bar{x}$ and $\underline{x}$ overlap, then it is an optimal solution of the QFP problem (1), and STOP.

- $\quad$ Else, assign $\underline{x}$ to $\bar{x}$, and go to Step 4 . 
The flow chart of the proposed approach is illustrated in Figure 1.

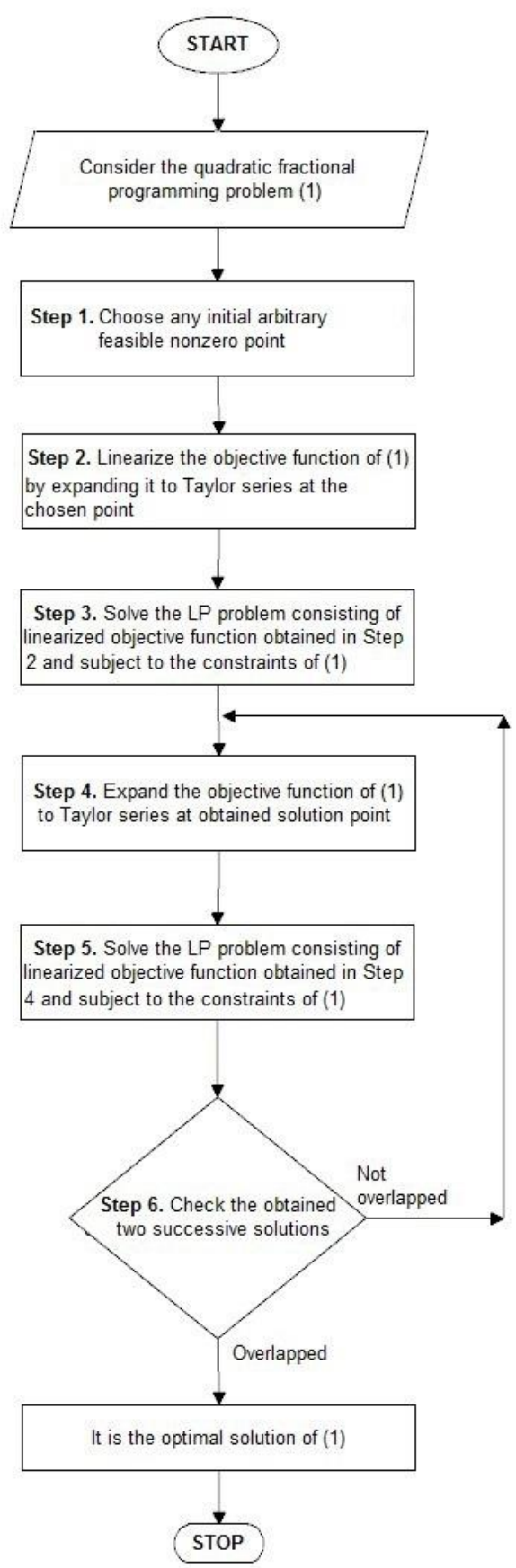

Figure 1: The flow chart of the proposed approach 
Different methods are compared with the proposed approach in Example 1 and Example 2. Table 1 and Table 2 show the results that are obtained by using our approach and the existing methods from the literature.

\section{Numerical examples}

Example 1[11]. Solve the following QFP problem:

$$
\operatorname{Max} z=\frac{\left(x_{2}+1\right)\left(x_{1}+x_{2}+3\right)}{\left(x_{1}+4\right)\left(x_{1}+x_{2}+2\right)}
$$

subject to

$$
\begin{aligned}
& -x_{1}+x_{2} \leq 1 \\
& x_{1}+2 x_{2} \leq 7 \\
& x_{1} \geq 0, x_{2} \geq 0
\end{aligned}
$$

with the proposed approach.

Steps 1-2. Choose an initial arbitrary feasible non-zero point $(1,1)$, and linearize the objective function (2.a) by expanding it to the Taylor series at the chosen point:

$$
\begin{aligned}
& \frac{\partial z}{\partial x_{1}}=\frac{\left(x_{2}+1\right)\left(x_{1}+4\right)\left(x_{1}+x_{2}+2\right)-\left(x_{2}+1\right)\left(x_{1}+x_{2}+3\right)\left[\left(x_{1}+4\right)+\left(x_{1}+x_{2}+2\right)\right]}{\left(x_{1}+4\right)^{2}\left(x_{1}+x_{2}+2\right)^{2}} \\
& \frac{\partial z}{\partial x_{2}}=\frac{\left[\left(x_{2}+1\right)+\left(x_{1}+x_{2}+3\right)\right]\left(x_{1}+4\right)\left(x_{1}+x_{2}+2\right)-\left(x_{2}+1\right)\left(x_{1}+x_{2}+3\right)\left(x_{1}+4\right)}{\left(x_{1}+4\right)^{2}\left(x_{1}+x_{2}+2\right)^{2}} .
\end{aligned}
$$

Step 3. Construct an LP problem as follows:

$$
\operatorname{Max} z=-0.125 x_{1}+0.225 x_{2}
$$

subject to

$$
\begin{aligned}
& -x_{1}+x_{2} \leq 1 \\
& x_{1}+2 x_{2} \leq 7 \\
& x_{1} \geq 0, x_{2} \geq 0
\end{aligned}
$$

The optimal solution of the LP problem (4) is $\bar{x}_{1}=1.6667$ and $\bar{x}_{2}=2.6667$.

Steps 4-5. Expand the objective function (2.a) to the Taylor series at $\bar{x}=(1.6667,2.6667)$, and form the LP problem as follows:

$$
\operatorname{Max} z=-0.1483 x_{1}+0.1882 x_{2}
$$

subject to 
206

$$
\begin{array}{r}
-x_{1}+x_{2} \leq 1 \\
x_{1}+2 x_{2} \leq 7 \\
x_{1} \geq 0, x_{2} \geq 0
\end{array}
$$

The optimal solution of (5) is $\underline{x}_{1}=1.6667$ and $\underline{x}_{2}=2.6667$.

Step 6. Because the two successive solutions, $\bar{x}$ and $\underline{x}$, overlap, the optimal solution of (2) is found to be $x_{1}^{*}=1.6667, x_{2}^{*}=2.6667$, and the optimal value is $z^{*}=0.7492$.

The proposed approach can be applied to the problem solved by Jayalakshmi [11], and it is seen that the solutions are identical. A comparison of the solutions obtained is presented in Table 1.

\begin{tabular}{|c|c|c|}
\hline & Jayalakshmi's Approach & Proposed Approach \\
\hline$x_{1}^{*}$ & 1.6667 & 1.6667 \\
\hline$x_{2}^{*}$ & 2.6667 & 2.6667 \\
\hline$z^{*}$ & 0.7492 & 0.7492 \\
\hline
\end{tabular}

Table 1: Comparison of the solutions obtained for Example 1

Example 2 [12]. Solve the following QFP problem:

$$
\operatorname{Max} z=\frac{\left(2 x_{1}+2 x_{2}+12\right)\left(x_{1}+x_{2}+17\right)}{\left(-2 x_{1}+x_{2}+15\right)\left(2 x_{1}-x_{2}+11\right)}
$$

subject to

$$
\begin{gathered}
x_{1}+2 x_{2} \leq 2 \\
3 x_{1}+x_{2} \leq 4 \\
-5 x_{1}+3 x_{2} \leq 0 \\
-x_{1}+x_{2} \leq 0
\end{gathered}
$$

with the proposed approach.

Steps 1-2. Choose an initial arbitrary feasible non-zero point $(1,1)$, and linearize the objective function (6.a) by expanding it to the Taylor series at the chosen point.

Step 3. Construct an LP problem as:

$$
\operatorname{Max} z=0.2783 x_{1}+0.343 x_{2}
$$

subject to

$$
\begin{array}{r}
x_{1}+2 x_{2} \leq 2 \\
3 x_{1}+x_{2} \leq 4 \\
-5 x_{1}+3 x_{2} \leq 0
\end{array}
$$




$$
-x_{1}+x_{2} \leq 0
$$

The optimal solution of ( 7$)$ is found to be $\bar{x}_{1}=1.2$ and $\bar{x}_{2}=0.4$.

Steps 4-5. Expand the objective function (6.a) to the Taylor series at $\bar{x}=(1.2,0.4)$, and form the LP problem as follows:

$$
\operatorname{Max} z=0.3673 x_{1}+0.3101 x_{2}
$$

subject to

$$
\begin{array}{r}
x_{1}+2 x_{2} \leq 2 \\
3 x_{1}+x_{2} \leq 4 \\
-5 x_{1}+3 x_{2} \leq 0 \\
-x_{1}+x_{2} \leq 0
\end{array}
$$

The optimal solution of (8) is $\underline{x}_{1}=1.2$ and $\underline{x}_{2}=0.4$.

Step 6. Because the two successive solutions, $\bar{x}$ and $\underline{x}$, overlap, the optimal solution of (6) is found to be $x_{1}^{*}=1.2, x_{2}^{*}=0.4$, and the optimal value is $z^{*}=1.6745$.

\begin{tabular}{|c|c|c|}
\hline & Khurana and Arora's Approach & Proposed Approach \\
\hline$x_{1}^{*}$ & 1.2 & 1.2 \\
\hline$x_{2}^{*}$ & 0.4 & 0.4 \\
\hline$z^{*}$ & 1.6745 & 1.6745 \\
\hline
\end{tabular}

Table 2: Comparison of the solutions obtained for Example 2

We apply the proposed approach to the problem given by Khurana and Arora [12], and the solutions obtained are the same as found by those authors. They are presented in Table 2 .

\section{Conclusion}

In this paper, a new approach is presented, based on solving LP problems to minimize the total execution time, which is useful for iterative operations. This approach can handle QFP problems having a factorized or non-factorized objective function, and homogenous or non-homogenous constraints. The proposed approach can easily be applied to solve any type of QFP problem. The QFP problem is converted into an LP problem with a chosen initial point. The proposed approach differs from other existing methods in terms of computational steps. It is also easier than other methods that can be solved algebraically. With the proposed approach, the final solution can be obtained rapidly, so it would be favorable for computer use. This approach can 
be implemented for various types of nonlinear FP problems containing nonlinear constraint(s) that are more complex.

\section{References}

[1] Abdulrahim, B. K. (2014). On extreme point quadratic fractional programming problem. Applied Mathematical Sciences, 8(6), 261-277.

[2] Bitran, G. R. and Magnanti, T. L. (1976). Duality and sensitivity analysis for fractional programs. Operations Research, 24(4), 675-699.

[3] Bitran, G. R. and Novaes, A. G. (1973). Linear programming with a fractional objective function. Operations Research, 21(1), 22-29.

[4] Charnes, A. and Cooper, W. W. (1962). Programming with linear fractional functionals. Naval Research Logistics (NRL), 9(3-4), 181-186.

[5] Chong, E. K. and Zak, S. H. (2013). An introduction to optimization. New York: John Wiley \& Sons.

[6] Effati, S. and Pakdaman, M. (2012). Solving the interval-valued linear fractional programming problem. American Journal of Computational Mathematics, 2(1), 51-55.

[7] Goshu, N. N. (2014). Fractional programming. International Journal of Engineering Science and Advanced Technology, 4(4), 323-339.

[8] Gotoh, J. Y. and Konno, H. (2001). Maximization of the ratio of two convex quadratic functions over a polytope. Computational Optimization and Applications, 20(1), 43-60.

[9] Hasan, M. R. and Hasan, M. B. (2014). An alternative method for solving quadratic fractional programming problems with homogenous constraints. Journal of Emerging Trends in Engineering and Applied Sciences, 5(1), 11-19.

[10] Isbell, J. R. and Marlow, W. H. (1956). Attrition games. Naval Research Logistics, 3(1-2), 71-94.

[11] Jayalakshmi, M. (2015). A new approach for solving quadratic fractional programming problems. International Journal of Applied Research, 1(9), 788-792.

[12] Khurana, A. and Arora, S. R. (2011). An algorithm for solving quadratic fractional program with linear homogeneous constraints. Vietnam Journal of Mathematics, 39(4), 391-404.

[13] Nguyen, V. B., Sheu, R. L., and Xia, Y. (2014). An SDP approach for solving quadratic fractional programming problems. arXiv preprint arXiv:1402.4198.

[14] Odior, A. O. (2012). An approach for solving linear fractional programming problems. International Journal of Engineering \& Technology, 1(4), 298-304.

[15] Pandian, P. and Jayalakshmi, M. (2013). On solving linear fractional programming problems. Modern Applied Science, 7(6), 90-100. 
[16] Pramanik, S., Dey, P. P. and Giri, B. C. (2011). Multi-objective linear plus linear fractional programming problem based on Taylor series approximation. International Journal of Computer Applications, 32(8), 61-68.

[17] Saha, S. K., Hossain, M. R., Uddin, M. K. and Mondal, R. N. (2015). A new approach of solving linear fractional programming problem (LFP) by using computer algorithm. Open Journal of Optimization, 4(03), 74-86.

[18] Schaible, S. and Shi, J. (2004). Recent developments in fractional programming: Singleratio and max-min Case. Nonlinear Analysis and Convex Analysis, 493-506.

[19] Sharma, K. C. and Singh, J. (2013). Solution methods for linear factorized quadratic optimization and quadratic fractional optimization problem. Journal of Mathematics, $8(3)$, 81-86.

[20] Simi, F. A. and Talukder, M. S. (2017). A new approach for solving linear fractional programming problems with duality concept. Open Journal of Optimization, 6(01), 1-10.

[21] Singh, C. (1981). Optimality conditions in fractional programming. Journal of Optimization Theory and Applications, 33(2), 287-294.

[22] Suleiman, N. A. and Nawkhass, M. A. (2013). A new modified simplex method to solve quadratic fractional programming problem and compared it to a traditional simplex method by using pseudoaffinity of quadratic fractional functions. Applied Mathematical Sciences, 7(76), 3749-3764.

[23] Swarup, K. (1965). Letter to the Editor - Linear fractional functionals programming. Operations Research, 13(6), 1029-1036.

[24] Tantawy, S. (2008a). An iterative method for solving linear fraction programming (LFP) problem with sensitivity analysis. Mathematical and Computational Applications, 13(3), $147-151$.

[25] Tantawy, S. F. (2008b). A new procedure for solving linear fractional programming problems. Mathematical and Computer Modelling, 48(5-6), 969-973.

[26] Tantawy, S. F. (2014). A new concept of duality for linear fractional programming problems. International Journal of Engineering and Innovative Technology, 3, 147-149.

[27] Tantawy, S. F. and Sallam, R. H. (2013). Research article: A new method for solving integer linear fractional programming problems. International Journal of Recent Scientific Research, 4(3), 250-253.

[28] Wolf, H. (1985). A parametric method for solving the linear fractional programming problem. Operations Research, 33(4), 835-841.

[29] Youness, E. A., Maaty, M. A. and Eldidamony, H. A. (2016). A two-dimensional approach for finding solutions of nonlinear fractional programming problems. Journal of Computer Science Approaches, 2(1), 6-10. 